\title{
Comerciar sin afiebrarse. Experiencias sensoriales y oposiciones cualitativas en la formalización de la economía esmeraldera en Colombia
}

\author{
Trade without Fever. Sensory Experiences \\ and Qualitative Oppositions in the Formalization \\ of the Emerald Economy in Colombia \\ Vladimir Caraballo Acuña* \\ Universidad Nacional de Colombia, El Colegio de Michoacán
}

\begin{abstract}
RESUMEN
Desde hace algunos años la explotación y el comercio de minerales en Colombia atraviesan un lento proceso de formalización en manos del Estado. En el caso de las esmeraldas, ha estado acompañado por la consolidación del Grupo Muzo, compañía estadounidense encargada de explotar, tallar y exportar las esmeraldas. En este artículo sugiero que ambos procesos han implicado la construcción de una ideología semiótica que organiza experiencias sensoriales de la economía esmeraldera alrededor de tres pares de cualidades opuestas: sucio/limpio, caliente/frío y opaco/ transparente. Para desarrollar la relación entre experiencias sensoriales e ideología propongo un acercamiento metodológico que combina los insumos de mi trabajo de campo y la antropología semiótica contemporánea, particularmente, aquella que recupera el pragmatismo filosófico de Charles Peirce.
\end{abstract}

Palabras clave: esmeraldas, formalización, experiencias sensoriales, ideología semiótica.

\begin{abstract}
For some years the mining and mineral trade in Colombia have undergone a slow process of formalization by the State. In the case of emeralds, this process has been accompanied by the consolidation of Grupo Muzo, the US company responsible for exploiting, carving, and exporting emeralds. In this article I suggest that both processes involve the construction of a semiotic ideology that organizes sensory experiences of the emerald economy around three pairs of opposing qualities: dirty/ clean, hot/cold, and opaque/transparent. To develop the relationship between sensory experiences and ideology, I suggest a methodological approach that merges inputs from my fieldwork and contemporary semiotic anthropology, particularly that which recovers the philosophical pragmatism of Charles Peirce.
\end{abstract}

Keywords: emeralds, formalization, sensory experiences, semiotic ideology.

\footnotetext{
Magíster en Antropología Social de El Colegio de Michoacán y doctorando en Antropología Social de la misma institución. Sus publicaciones incluyen "Órdenes locales, acuerdos de paz y presencia diferenciada del Estado. Negociación con las Milicias Populares de Medellín”, Colombia Internacional 77 (2013): 241-270. mvacuna@colmich.edu.mx.
} 


\section{Introducción}

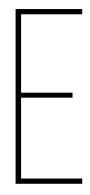

n mayo del 2015, decenas de mineros decidieron tomarse por la fuerza uno de los cortes esmeralderos administrados por la empresa estadounidense Minería Texas Colombia (MTC) en el municipio de Muzo, al occidente de Boyacá. Al parecer, se había regado el rumor de una nueva y productiva veta y fue cuestión de horas para que los mineros se organizaran, cortaran las rejas, tumbaran las puertas y entraran a buscar esmeraldas ("Polémica tras la venta más grande de Carranza” 2015). MTC hace parte del Grupo Muzo (en adelante GM), una compañía estadounidense filial de Texma Group, constituida por dos empresas más: Colombiano Texas Transformadora (CTT) —encargada de tallar las piedras_y Muzo International —encargada de su comercialización fuera de Colombia-. MTC fue la primera de las tres en constituirse en el año 2009 gracias a la relación que Charles Burgess, su presidente, había logrado construir con el fallecido Víctor Carranza (Blanco 2015). En ese momento, la compañía se hizo con un porcentaje de las acciones de Carranza y sus asociados - hasta entonces encargados de la administración de varios cortes, principalmente de la mina Puerto Arturo, una de las más productivas del mundo-y en el 2013 logró adquirir su totalidad. Dos años después, con la extracción, la talla y la comercialización aseguradas, el GM hizo una cena como lanzamiento oficial en el Museo Botero de Bogotá (Pérez 2016).

En el 2015, unos días después de la toma, Charles Burgess declaró lo siguiente:

Quiero recordar a los colombianos que estos son bienes del Estado, y por lo tanto la Compañía tiene que pagar regalías e impuestos. Quienes están sacando las esmeraldas, las están entregando a gente que no paga regalías ni impuestos, y que exportan las esmeraldas de manera ilegal en el país. Yo creo que ningún colombiano puede apoyar esa clase de conducta [...] En la mina y en la zona queremos relaciones formales, legales con todo el mundo. Hacemos todo de acuerdo con la ley. Tenemos

Este artículo corresponde a un avance de investigación en el marco del Doctorado en Antropología que curso en El Colegio de Michoacán, México. El trabajo de campo se ha llevado a cabo en los distintos circuitos del comercio de esmeraldas a partir de mi participación como tallador y comerciante en Bogotá y de cortas estadías en el occidente de Boyacá desde el 2014. Un reporte de estos avances fue presentado en noviembre del 2016 en el encuentro anual de la American Anthropological Association en Minneapolis. Agradezco a los comerciantes, comisionistas, mineros, talladores y gemólogos que han colaborado en el desarrollo de esta investigación. También a Brian Brazeal, Mauricio Montenegro, Daniel Ramírez y Ana María Restrepo por sus comentarios sobre el primer manuscrito. De igual manera, al Consejo Nacional de Ciencia y Tecnología de México y al Consejo Latinoamericano de Ciencias Sociales por los recursos otorgados. 
una cultura basada en el respeto de nuestros trabajadores y el buen trato. Somos tan legales, que hasta tenemos sindicato. Queremos relaciones que nos permitan cambiar las reglas arcaicas y violentas que han imperado en esta zona. Es como si Cerrejón o Drummond encuentran una nueva veta y los vecinos, acostumbrados a vivir bajo unas reglas arbitrarias, deciden invadir la mina y explotarla a causa propia, sin que el Estado haga nada. Yo entiendo los problemas de pobreza y los sueños de riqueza que tienen, pero la verdad es que la idea de que van a cambiar su vida arriesgando en una mina, buscando unas piedras, no es más que una simple fábula. ("Minería Texas Colombia” 2015; "Polémica tras la venta más grande de Carranza” 2015)

En la actualidad, el GM ha contratado formalmente a cerca de setecientos empleados -entre personal de las minas en Muzo, talladores en Bogotá y personal administrativo-, ha invertido millones de dólares en la tecnificación de la explotación, ha implementado nuevas e intensas técnicas de seguridad en las minas para evitar los robos y, ante todo, ha creado un circuito propio para la explotación, la talla y la venta de las esmeraldas. Las piedras que extrae MTC viajan en helicóptero desde Muzo y en Bogotá son valoradas para el pago de los respectivos impuestos. Luego, la compañía las transporta a la Zona Franca en donde son talladas por los talladores colombianos de CTT para, finalmente, ser enviadas al exterior. Las esmeraldas extraídas por la compañía circulan de manera absolutamente controlada y privada, es decir, eludiendo el mercado tradicional, en particular a los comerciantes y comisionistas congregados en el centro de Bogotá y en las “playas” (lugares de comercio local) de la región minera. Por eso, los comerciantes del centro coinciden en que la llegada de la compañía ha implicado la creciente escasez de las piedras.

La presencia del GM se ha dado de forma simultánea a un lento proceso de formalización minera en manos del Estado colombiano. La formalización busca encarrilar la explotación y el comercio de minerales en dos lógicas del mercado global: la transparencia y la trazabilidad. La larga lista de normas orientadas a la formalización incluye dos declaraciones centrales, el artículo 112 de la Ley 1450 del 2011 y el Decreto 2637 del 2012. El primero decretó que, en adelante, "los compradores y comercializadores de minerales solo podrán adquirir estos productos a los explotadores y comercializadores mineros registrados” y que, de lo contrario, se decomisará el mineral no acreditado y se estipulará la multa correspondiente. El segundo dispuso los instrumentos necesarios para dichos registros, especialmente el Registro Único de Comercializadores de Minerales (Rucom) y el Certificado de Origen, además de los exigidos a cualquier persona o entidad comercial para sustentar su funcionamiento financiero. El Rucom es: 
[...] el registro único de comercialización de minerales, en el cual deberán inscribirse los titulares de derechos mineros que se encuentren en etapa de explotación y que cuenten con las autorizaciones o licencias ambientales requeridos, así como los agentes comercializadores de minerales como requisito para tener acceso a la compra y/o venta de minerales. (Decreto 2637 del 2012)

Como complemento, el Certificado de Origen está orientado al registro de los minerales:

Es el documento que se emite para certificar la procedencia del mineral en el que conste la fecha y el lugar de la producción de minerales que se transporten, transformen, distribuyan, intermedien o comercialicen, expedido por el titular minero; por la alcaldía, cuando se trate de minerales productos de barequeo [guaqueo], o por la Agencia Nacional de Minería, en el caso de solicitudes de legalización en trámite y beneficiarios de áreas de reserva especial. (Decreto 2637 del 2012)

Mediante estos documentos, el Estado colombiano pretende construir un sistema de legibilidad de mineros, comerciantes y minerales, garantizar el pago de los impuestos respectivos y avanzar en la competitividad del mercado colombiano en torno al llamado mine-to-market, es decir, con respecto a la posibilidad de que los consumidores "finales" conozcan las condiciones de explotación y comercio de minerales como las esmeraldas. Al igual que la presencia de MTC en la región minera del occidente de Boyacá, la formalización no ha sido ajena a luchas políticas. El 18 de febrero del 2015, cerca de quinientos comerciantes, comisionistas y talladores de esmeraldas marcharon desde la plaza del Rosario hasta la plaza de Bolívar en Bogotá. Las consignas de la marcha hablaban de la nueva legislación, de la presencia de compañías multinacionales y del abandono de los municipios mineros de parte del Estado. En una de las pancartas se leía: "Antes los españoles se llevaron nuestras riquezas... Hoy la locomotora minera de [el presidente] Santos ${ }^{2}$ se las entrega a las multinacionales. No al abuso del Gobierno. Los mineros, comisionistas y comerciantes de esmeraldas, Zona Centro" (Vega 2015). Muchos dicen que no están dispuestos a pagar impuestos que disminuirían las pocas ganancias que obtienen en un escenario de escasez generada, en parte, por el circuito privado del GM. Otros dicen que una verdadera formalización implicaría la formación profesional de los comisionistas y comerciantes,

2 Locomotora minera fue la expresión que el presidente usó para referirse a la continuación de las políticas orientadas al crecimiento exponencial de la explotación minera en el país, particularmente, en manos de agentes privados. Dichas políticas han implicado complejos conflictos en el marco de los cuales toma forma este artículo. 
y no solo la exigencia del pago de impuestos. Otros no aceptan que se les exija a ellos mientras que se abren las puertas al enriquecimiento de la compañía extranjera.

En este artículo sugiero que si bien el proceso de formalización y la presencia del GM representan un conflicto por la extracción y circulación de un recurso valioso en los términos en los que los conflictos mineros suelen ser analizados (Ballard y Banks 2003; Gutiérrez y Barón 2008; Leiteritz y Riaño 2016), es necesario ampliar la mirada para preguntar no solo por la extracción del valor, sino por la creación de este. En otras palabras, las esmeraldas no son objetos valiosos en sí mismos; más bien, hacerlos valiosos implica históricos y complejos ensamblajes económicos, sociales y políticos cuyos desarrollos deben ser explicados (Ferry y Limbert 2008). Con el enfoque semiótico que propongo, busco centrar la atención en pequeñas y representativas prácticas de construcción del valor de esmeraldas, personas y espacios, a través de la construcción y distribución de signos y tipos sociales. Además de pretender acercar los estudios clásicos sobre minería a los dedicados a la construcción de valor, este enfoque me permite no solo avanzar en análisis detallados de dicha construcción en el mundo de los minerales, sino poner en cuestión la aparente oposición entre dos parejas: economía política / análisis macrosociales y semiótica / análisis microsociales (Carr y Lempert 2016, 8). En este escrito pretendo abonar a esta discusión de larga data que, sin embargo, supera los objetivos del artículo ${ }^{3}$. Concretamente, muestro la construcción de una ideología semiótica que organiza experiencias sensoriales propias del comercio tradicional en una escala de valor orientada por la transparencia y la trazabilidad como estándares del comercio global. Argumento que, como se verá a partir de ejemplos etnográficos concretos, en esta ideología dichas experiencias son leídas desde tres pares de cualidades opuestas: sucio/limpio, caliente/frío y opaco/transparente. A pesar de que sus impulsores la presentan como un proceso inevitable de modernización de la minería, propongo entenderla, más bien, como un conjunto de recursos simbólicos invocados de manera creativa en prácticas específicas. Para sustentar mi argumento acudo a información de mi trabajo de campo realizado en el comercio bogotano y en la región minera del occidente de Boyacá, y dialogo con estudios contemporáneos que recuperan las propuestas del filósofo estadounidense Charles Sanders Peirce. Antes de entrar de lleno al desarrollo del argumento, dedicaré un par de páginas a una muy resumida - y por lo mismo, densa- presentación teórica de las propuestas peirceanas recuperadas recientemente en la antropología; en un

3 Algunos acercamientos significativos pueden ser leídos en Ferry (2016a, 2016b); Gal (1989); Keane (2003); Kockelman (2016, 2006); Maurer (2006). 
segundo momento, organizo algunas experiencias etnográficas alrededor de la suciedad, de lo caliente y de lo opaco para, por último, mostrar cómo estas experiencias cualitativas del comercio y la explotación tradicionales han comenzado a ser organizadas en una ideología que las dota de nuevos significados, en una escala de valor propia de la formalización minera.

\section{Qualias y cualisignos: de las experiencias sensoriales a las convenciones sociales}

En los últimos años, gracias a los trabajos pioneros de Nancy Munn (1986) y Michael Silverstein (1976), algunos antropólogos se han dedicado al desarrollo de las propuestas semióticas de Charles Peirce (1839-1914), en torno a una preocupación común: explicar los procesos sociales por los cuales se construye la relación entre experiencias sensoriales específicas y convenciones sociales de más largo alcance. En términos metodológicos, se han preguntado qué nos dicen experiencias sensoriales concretas - de la voz suave de un cantante, de las cualidades materiales de un diamante o del color gris de la fachada de un edificioacerca de sistemas de representación temporal y espacialmente más amplios. En este camino se encuentra el estudio de Nicholas Harkness (2014) acerca de las cualidades sensoriales de la voz en la modernización de Corea; el de Krisztina Féherváry (2013) sobre el papel del color gris en la formación de la clase media de la Hungría postsocialista; la propuesta de Lily Chumley (2013) para entender la relación entre experiencias sensoriales y regímenes de evaluación en escuelas de arte en China; el trabajo de Daniel Valentin (1996) sobre la relación semiótica entre tortura y belleza en episodios violentos de Sri Lanka; el de Filipe Calvão (2013), en el que analiza la velocidad y la lentitud como sensaciones del comercio de diamantes en Angola, y la adjudicación de cualidades sensoriales a las palabras en relación con ideologías lingüísticas en Hungría, de parte de Susan Gal (2013), entre otros.

La relación entre experiencias sensoriales y concepciones abstractas encontraba ya lugar en los desarrollos de Peirce, fundador del pragmatismo estadounidense del siglo XIX. En palabras de Sara Barrena (2014), filósofa española dedicada a su estudio:

Para Peirce, nuestra idea de algo es nuestra idea de sus efectos sensibles, y el significado de una concepción viene determinado por las consecuencias prácticas de esa concepción. El reconocer un concepto 
bajo sus distintos disfraces o el mero análisis lógico no es suficiente para su comprensión, sino que es necesario alcanzar un tercer grado de claridad que solo puede obtenerse a través de los efectos prácticos del concepto. (4)

Para los pragmatistas, la única forma de entender el conocimiento, las ideas, era entender sus consecuencias prácticas, experimentadas. En este camino, Peirce buscó construir un sistema lógico que permitiera demostrar no solo la articulación entre experiencias sensoriales e ideas abstractas sino, ante todo, la mediación de las convenciones en esta relación. Para ello, ingenió un conjunto de tricotomías semióticas en las que la relación entre lo que llamó primeridad (entidades abstractas y potenciales) y segundidad (las entidades experimentadas) tenía lugar gracias a un tercer elemento, terceridad, que daba forma convencional (aunque no arbitraria) a la relación. Por este camino, Peirce rescataba cinco aspectos en la construcción del conocimiento: su naturaleza experimental o pragmática, el énfasis en las cualidades, la importancia del mundo sensorial, la construcción de convenciones o hábitos y, por último, su carácter procesual, en permanente elaboración.

Partiendo de allí, los análisis antropológicos contemporáneos han buscado desarrollar lo que Nicholas Harkness (2015) llama la pragmática sensorial de la práctica o los procesos a través de los cuales las prácticas sensoriales son tipificadas, situadas y significadas (583). Entre las muchas tricotomías lógicas en las que tienen lugar la primeridad, la segundidad y la terceridad (varias de las cuales se pueden leer en Restrepo [1990]), algunos de estos antropólogos han avanzado en el desarrollo de una en particular: cualidad (primeridad), qualia (segundidad) y cualisigno (terceridad). La relación entre cualidades abstractas y qualias es descrita por Harkness (2013) de la siguiente manera:

La experiencia cualitativa está hecha de lo que Peirce llama qualia, las instancias realizadas de cualidades que son moduladas por, y relacionadas con, el pensamiento, la materialidad, los canales sensoriales, etc. Las qualias son vividas. [...] La abstracción de las cualidades es experimentada y conocida a través de qualia (p. ej. la "rojedad" abstracta versus la "rojedad" de la piel de una manzana particular). (14) (Todas las traducciones son propias)

En primer lugar, las qualias son experiencias sensoriales que remiten a cualidades abstractas como la suavidad de un trago de soju ${ }^{4}$, la suciedad del trabajo minero, la rugosidad de la voz de alguien en una conversación, etc. En 
segundo lugar, esas experiencias no se estancan en lo subjetivo, en las entidades concretas que las viven; más bien, son construidas intersubjetivamente. En tercer lugar, compartidas, las qualias se convierten en puntos de orientación o coordenadas que las personas utilizan para relacionarse, así, la suavidad, la suciedad o la rugosidad trascienden las múltiples experiencias en las que pueden darse, y se convierten en convenciones que pueden constituir regímenes de valor, es decir, son evaluadas y, a la vez, son utilizadas para evaluar otras experiencias y a otras personas (Chumley 2013, 169). Este tercer punto es quizá el más importante, cuando experiencias sensoriales de distinto tipo comienzan a empaquetarse como modalidades de una misma cualidad (por ejemplo, en el caso de Harkness, la suavidad de un trago de soju y la suavidad de las relaciones en una fiesta juvenil), y cuando entonces "lo suave" empieza a convertirse en una convención que orienta las prácticas discursivas y no discursivas (en el mismo ejemplo, la suavidad como una cualidad impulsada por los discursos acerca de la modernización de Corea), es posible hablar de ellas como cualisignos. En términos peirceanos, los cualisignos son signos de la terceridad que permiten que experiencias sensoriales de distinto tipo (qualias) se asuman como instancias de una misma cualidad (primeridad) y que dan a esta relación la forma de una convención. La naturaleza convencional de los cualisignos permite que las experiencias sensoriales se conviertan en coordenadas de orientación social y, de allí, a través de procesos sociales específicos, en regímenes de valor a través de los cuales los actores distribuyen personas y objetos en el mundo social. Desde Peirce hasta sus desarrollos contemporáneos en la antropología, la preocupación pareciera ser, hasta cierto punto, similar. Chumley y Harkness (2013) la resumen en la introducción del número 1 de Anthropological Theory dedicada al concepto de qualia:

Los artículos de este número ofrecen claves etnográficas de las condiciones discursivas, históricas y políticas bajo las cuales las sensaciones comienzan a ser entendidas como sensaciones de cualidades - la qualia de la suavidad, la luminosidad, la transparencia, el dolor, el mal olor, etc.- y en las cuales esas qualias son articuladas con valores culturales, bien sean positivos o negativos. (13)

Dicho lo anterior, quisiera ahora organizar algunas experiencias propias del comercio tradicional a partir del concepto de qualia para, posteriormente, ir mostrando cómo han comenzado a organizarse en lo que puede ser entendido como el carácter semiótico de la formalización minera. 


\section{Qualias y cualisignos de la economía esmeraldera}

Lo limpio y lo sucio son formas de referirse al grado de inclusiones y "negritos" de una esmeralda. Las inclusiones son sustancias en forma líquida, sólida y gaseosa que forman parte de la morfología de las esmeraldas. Dependiendo de sus tamaños, constituciones y distribuciones, estas inclusiones pueden hacer que las esmeraldas refracten de mejor o peor manera la luz que es, en últimas, la principal cualidad de las piedras: refractar la luz. Cuando las inclusiones impiden que la luz circule en el interior de la piedra, para luego regresar a los ojos de quien entonces la ve cargada de color verde, esas inclusiones (o "negritos") hacen que la esmeralda se vea sucia. La suciedad puede ser también causada por “nubes”, por "gasas" o por fisuras que generan el mismo efecto. Pero, además de ser atributos asignados a las esmeraldas, lo limpio y lo sucio hacen parte de su proceso de extracción. Estar sucio es una experiencia sensorial propia del trabajo minero. Específicamente, es estarlo con el barro de los túneles mineros, aunque el grado de suciedad depende de las funciones en la mina (se ensucia menos el encargado de operar el ascensor que el de picar la roca) y del tipo de mina. Durante mi trabajo de campo, ingresé varias veces a túneles artesanales que en la región reciben el nombre de voladoras. Allí, la tecnología de enfriamiento es realmente precaria, no hay señales de orientación, no hay luces (excepto la del casco), la oscuridad es casi absoluta y el calor, infernal. De allí, sea cual sea la función, se sale absolutamente empapado y embarrado. Pero lo importante es que, en tanto qualia, la suciedad no es solo esa experiencia sino el objeto de valoración y, al mismo tiempo, un medio para valorar. Veamos. Hilda $\mathrm{Cruz}^{5}$, integrante de la Confederación de Esmeralderos de Colombia (Confedesmeraldas), lo tiene claro. La Confedesmeraldas nació en el 2014 como una suerte de disidencia de la Federación Nacional de Esmeraldas de Colombia (Fedesmeraldas), entidad mixta (estatal y privada) que administra las contribuciones parafiscales pagadas por el gremio ${ }^{6}$. En marzo del 2016 pude hablar con varios de sus integrantes acerca de un proyecto de valor que consiste, principalmente, en conseguir que las

5 Todos los nombres y seudónimos de los colaboradores de esta investigación han sido cambiados por seudónimos (en el primer caso) y por seudónimos de seudónimos (en el segundo).

$6 \quad$ Las contribuciones parafiscales se refieren al gravamen que un gremio específico ha decidido establecer a sus propios integrantes y que, aunque administradas de manera mixta (privada y estatal), son de utilidad exclusiva del gremio, y no de la totalidad de la Nación, como sí ocurre con los impuestos. 
esmeraldas sean declaradas "patrimonio cultural” y garantizar así, según dicen, que la riqueza se quede en el país y no en manos de compañías como el GM. Para construir su proyecto acuden a la ancestralidad, al lenguaje de patrimonio (Ferry 2011) y a la oposición sucio/limpio en la que quiero profundizar. Hilda dice con orgullo que (al igual que las esmeraldas) ella "salió 'dentre' el barro". Con ello no se refiere solamente a su antiguo trabajo como guaquera sino a haber crecido en la pobreza, a ser de la región minera y a ser campesina. En las historias que cuenta siempre hay alguien sucio que llega a pedir posada a una casa o alguien que llega embarrado de trabajar en las minas. En estas historias, la suciedad no es solamente la sensación concreta de tener barro en el cuerpo, sino una forma de ubicarse a sí misma y a otros en relaciones de poder particulares. Así, no solo diferencia a quienes se han embarrado de los que nunca lo han hecho, sino que además relaciona directamente la suciedad con el sufrimiento y con el conocimiento:

Se encuentra uno aquí [en Bogotá] con que lo tratan, por ser de allá del área [el occidente de Boyacá]: "sumercé escuche porque sumercé no sabe”. Y uno se sienta a escucharlos, iy nadie sabe la lógica de lo que ocurre! ¿Cuántos de estos grandes empresarios no se han embarrado los zapatos? Y yo no estoy en contra de ellos, sino... hombre... ellos no saben adónde va el dolor. (Confedesmeraldas, entrevista colectiva, marzo del 2016; énfasis añadido)

Para Hilda, la suciedad es una experiencia concreta pero, ante todo, es una experiencia que permite diferenciar a las personas en relación con lo que saben y el dolor que han tenido que atravesar. En sus palabras, simplemente hay lógicas que no pueden ser conocidas sin haberse ensuciado y sin haber sufrido. En sentido peirceano, la suciedad del trabajo minero no es solo una experiencia sensorial sino una convención que permite orientarse en conflictos particulares y que puede ser entendida como un cualisigno. Ahora bien, si, como dije al inicio, entrar a las minas implica ensuciarse, también de manera inevitable implica acalorarse. El calor de los túneles clandestinos es soportable apenas por unos minutos a riesgo de desmayarse; en las minas más tecnificadas ocurre algo similar aunque estas cuentan con mecanismos de ventilación que enfrían el ambiente. Pero el calor no es solo una cualidad de la extracción de las esmeraldas. Al igual que la suciedad, lo caliente ha terminado por referirse a múltiples experiencias que convergen en una expresión que durante décadas ha acompañado la economía esmeraldera, la fiebre verde.

La fiebre verde remite a tres experiencias sensoriales típicas de "lo caliente” en la explotación y el comercio de esmeraldas. En primer lugar, se refiere a las altas temperaturas en que las piedras deben ser buscadas, sobre todo, 
cuando se trata de modos de explotación en los que el enfriamiento se hace por medios artesanales. En segundo lugar, permite hablar de la búsqueda compulsiva, “afiebrada”, de las esmeraldas. Tercero, remite al calor que se siente en las negociaciones mismas de las piedras en un escenario que, como el tradicional, es incierto, rápido y riesgoso, y en el que se ponen en juego grandes cantidades de dinero. Además de ello, la expresión fiebre verde sirve para interpretar a las piedras como producto de alguna enfermedad que, como bien es sabido, puede llegar a obnubilar el pensamiento y a producir alucinaciones. Cuando inicié la investigación en la que se basa este artículo me interesaba indagar por la fiebre verde. Tenía la sospecha de que la creencia en el "embrujo" que causaban las esmeraldas estaba íntimamente relacionada con las lógicas de producción y de regulación que, centradas en la suerte, los riesgos y la incertidumbre, ofrecían las condiciones de posibilidad para la emergencia y el sostenimiento de esos discursos. Sin embargo, durante el trabajo de campo fui viendo que ya nadie hablaba de la fiebre verde o que, más bien, hablaban de ella como un asunto del pasado. La explicación que me daban los mineros y comerciantes se refería a un asunto principal, el fin del boom esmeraldero, el aumento de la escasez. La fiebre verde, la obsesión por conseguir las esmeraldas en condiciones precarias que en no pocas ocasiones ponían en juego la vida propia y la de los otros ${ }^{7}$, era la herramienta lingüística con la cual se leía la abundancia. La transición de la explotación a cielo abierto hacia la explotación por túneles en la década de los noventa había sido originada, justamente, por la escasez de las piedras que antes se

Prácticamente, toda la literatura académica y no académica ha versado sobre el periodo de las guerras verdes. Aquí no profundizo en ello. Un reciente estado de la cuestión fue elaborado por Brian Brazeal $(2014,2016)$. Entre las investigaciones pioneras sobre ese periodo se encuentran las de Guerrero (1985), Páramo (2011) y Uribe (1992); también las de Claver (1993), Parra (2006), Pineda (2008), Piñeros y Herrera (2004), Restrepo, Polo y Mayorga (1996), y Torres y Ordóñez (1995). Una reciente actualización periodística fue elaborada por Petrit Baquero (2017). En proceso de publicación está la propuesta de Ralf Leiteritz y Manuel Riaño (2016) que analiza la relación entre las esmeraldas, el narcotráfico y la intensidad de la violencia en la región con énfasis cuantitativo. Aunque este reciente texto ofrece un panorama importante sobre la explotación de las esmeraldas y sobre algunos cambios recientes, llama la atención que no mencione las consecuencias de la formalización minera y, sobre todo, que haga uso de la oposición formal/informal y de adjetivos como ilegal, clandestina o arcaico para referirse a prácticas tradicionales, sin reflexionar acerca del uso político de estas categorías en el momento actual. Además, los autores conciben los "recursos naturales" como objetos dados en sí mismos, distanciándose con ello de la concepción más compleja a la que aquí me adhiero (véase Ferry y Limbert 2008). Por último, recientemente la revista Semana ha publicado un amplio especial dedicado al estado actual de la economía esmeraldera (http:// www.semana.com/seccion/contenidos-editoriales/esmeraldas-historias-por-contar-/384). Su énfasis está puesto en las ventajas de la formalización y de la presencia del Grupo Muzo. Vale la pena decir que se hace poca o ninguna mención a los conflictos violentos con MTC en la región, a las marchas de comerciantes en Bogotá, a los debates acerca de la exportación de esmeraldas en bruto (no talladas) de parte del grupo (véase la página 29 de este artículo) o al papel de Confedesmeraldas, organización opuesta tanto a la formalización como a la presencia del grupo. 
encontraban en la superficie, arando la tierra, en el interior de una gallina lista para cocinar o simplemente caminando por los terrosos caminos del occidente boyacense. Esta escasez inicial de esmeraldas se ampliaría con el cambio en la forma de buscarlas: el tambre - la tierra sobrante de las exploraciones mineras que era arrojada a las aguas del río Minero para el sustento de los guaqueros y, con ello, para el sustento de gran parte del comercio tradicional en la región y en Bogotá- había disminuido debido a la explotación por túneles. A esto se sumaban la presencia del GM y políticas ambientales más recientes que prohíben la contaminación de las aguas con el tambre. Por ello, en el comercio del centro de Bogotá todos coinciden en que la abundancia ha desaparecido y, con ella, la fiebre verde. Un reporte del 2011 en el diario El Espectador cita a un poblador de Muzo hablando del pasado boyante:

Alberto Pachón, propietario del Hotel El Castillo recuerda: "No podíamos dormir, había que atender a la gente a toda hora, sobre todo los fines de semana. Teníamos muchas meseras, dos chefs, empleadas para las habitaciones... Los manes llegaban y querían una botella de aguardiente, no les importaba el precio; ochenta mil, cien mil pesos... ‘Démela!', decían. Unos dormían en colchones en el suelo, porque las habitaciones estaban llenas. Descansaban un par de horas y volvían a la quebrada para guaquear”. El lugar hoy casi vacío es enorme. Las salas están desiertas y guardados en los rincones los refrigeradores industriales y el equipo de música. Las mesas, apiladas. "Las calles vivían llenas de gente”, añade, nostálgico. (Dessay 2011)

La fiebre verde conjuga, entonces, experiencias sensoriales alrededor del calor y las remite a un pasado de abundancia. La permanencia de esta expresión que durante décadas ha representado la economía esmeraldera tiene que ver justamente con que en ella convergen múltiples experiencias sensoriales que, reconocidas por todos, terminan convirtiéndose en una convención. Lo caliente - por vía de novelas, telenovelas, documentales en YouTube (Páramo 2011) — se convierte en un cualisigno de la economía esmeraldera, en una metáfora que se hace literal en experiencias concretas alrededor de la circulación de las piedras.

Hay un tercer conjunto de experiencias propias del comercio de esmeraldas, cuya aglutinación ideológica en la cualidad de "lo opaco" ha comenzado a formarse en los últimos años. Me refiero a un grupo de prácticas - particularmente, el uso de apodos, las múltiples relaciones cara a cara que dificultan el seguimiento de las piedras desde su salida de la mina hasta su venta "final" y los esfuerzos especulativos para fijar los precios de las piedras- que propongo ver, más bien, como formas de orientación en un escenario muy similar a lo que alguna vez Clifford Geertz (1978, 29-31) describió para las economías de bazar, poco 
reguladas, multidimensionales (en donde no solo se negocia monetariamente) y en donde el control de la información es central. Son justamente las dimensiones no monetarias (la reputación, la confianza, las relaciones de patronazgo, las redes familiares o de amistad, el prestigio, el carisma, el buen humor) las que hacen que el mercado esmeraldero tradicional sea tan intrincado y que los mecanismos de búsqueda y protección de la información sean tan densos como naturalizados por sus actores. De cierta manera - quiero argumentar aquí-, estas prácticas que han comenzado a organizarse en torno a la cualidad de "lo opaco", como mostraré en el siguiente apartado, son en realidad coordenadas de orientación en el comercio tradicional. En efecto, el comercio tradicional de esmeraldas en Colombia gira alrededor de la confianza (Brazeal 2015), de negociaciones que solo pueden tener lugar cara a cara, de relaciones de amistad, patronazgo y parentesco que llevan décadas consolidándose. En estas relaciones es poco usual que se conozcan los nombres. Al inicio de mi trabajo de campo insistía ingenuamente en conocer los nombres "verdaderos"; ante las reiteradas respuestas de mineros, talladores y comerciantes que confesaban la ignorancia al respecto, terminé entendiendo que los nombres “verdaderos” eran los apodos: Tripichín, Buche, Mentiras, Santoquemado, Gota, Puño, para no hablar de Orejas, de El Pequinés o de El Zar. Poco a poco, entonces, dejé de preguntar por ellos y opté por aprender los apodos. Una vez reconocida esta necesidad, la compleja red de comerciantes comenzaba a hacerse un poco más clara. Es justamente la complejidad de esa red la que hace difícil el seguimiento de la mayoría de las piedras. En algún momento me propuse hacerlo con una piedra cuyo destino “final” había sido una joyería en Manhattan. Aunque el exportador encargado de venderla a sus socios estadounidenses pudo dar cuenta de a quién se la había comprado, quién la había tallado, el laboratorio en donde la habían embellecido y certificado y los nombres de sus socios, no había manera de conocer cómo había llegado a su propietario anterior ni al consumidor final. El último punto fue imposible de dilucidar. En cuanto al primero, fueron los viajes a la región que hice en otros momentos y un par de llamadas telefónicas las que, luego de un buen tiempo, me permitieron establecer el total de catorce pares de manos por los que había pasado la piedra. Igual de difícil fue determinar los precios de cada momento de la circulación. Si cada quien conoce el apodo del propietario anterior de la piedra (con quien se realiza la negociación cara a cara), no ocurre lo mismo con los precios. Hasta cierto punto aquí se trata de algo oculto en dos sentidos: el primero tiene que ver con la materialidad misma de las piedras y el segundo, con la figura de las comisiones. En el primer sentido, una suerte de fatalidad material pareciera imposibilitar la formulación de precios 
estables en las esmeraldas, a diferencia de otras piedras preciosas. Todos con quienes he hablado - desde mineros hasta funcionarios de la Agencia Nacional Minera- coinciden en que, a pesar del establecimiento de las llamadas cuatro $c$ para la valoración de cualquier piedra preciosa (por las iniciales en inglés de las cualidades: color, cut, carat weight y clarity), la única manera de establecer el precio de una esmeralda es la experiencia, el regateo y el "precio de mercado". Tal como me dijera la misma Hilda: "el único lugar donde [la esmeralda] coge valor es en su mano" (Confedesmeraldas, entrevista colectiva, marzo del 2016). Las combinaciones entre el tipo de verde, su grado de saturación, su distribución en la piedra, la ubicación del cono luego de haber sido tallada, la calidad de las facetas talladas, la presencia, tipo y distribución de las inclusiones y el tamaño han impedido que los intentos de conmensuración de parte de entidades internacionales como la Confederación Internacional de Bisutería, Joyería y Orfebrería (Cibjo) o el Gemological Institute of America (GIA) sean acogidos. Sumado a esta dificultad en el establecimiento de los precios de las piedras está el asunto de las comisiones. Al lado de los comerciantes de esmeraldas se encuentra la figura del comisionista. Los comisionistas no son dueños de piedras. Su labor consiste en saber quién vende qué y quién está interesado en comprar qué; son intermediarios que viven de las comisiones obtenidas por una labor que representa de mejor manera el papel de la especulación ${ }^{8}$, del control de la información y del regateo. Lo curioso es que su intermediación implica, también, que usualmente ni el vendedor ni el comprador conozcan con certeza la comisión que está cobrando por intermediar. En otras palabras, la importante tarea del comisionista implica que, gran parte de las veces, el vendedor nunca sepa en cuánto compraron su piedra ni el comprador cuánto dinero recibió el dueño (véase Ferry, en prensa, cap. 6). Su función gira alrededor del conocimiento de las piedras pero, ante todo, del conocimiento de las personas, de sus intereses, de sus "modos de ser", como me dijera uno de ellos.

Esta serie de experiencias sensoriales alrededor de lo sucio, lo caliente, las negociaciones cara a cara, los esfuerzos implicados en descifrar los circuitos y las huellas de esmeraldas y de personas en el mercado ha comenzado a ser organizada en el marco del proyecto de valor que subyace a la formalización de la minería. Esta organización puede ser entendida como un proceso semiótico en el que las experiencias sensoriales concretas son leídas como signos en un régimen de valor formado por las tres oposiciones mencionadas. Lo sucio ya no sirve solamente para referirse al barro de las minas (como una qualia específica) sino 
para definir la tradición y el pasado del mercado como "sucios” en oposición a un mercado y un futuro "limpios"; lo caliente se convierte en una cualidad para referirse a la manera de comerciar de los mineros en oposición a quienes lo hacen con "cabeza fría”, sin afiebrarse, sin pasión, racionalmente. El uso de apodos, el papel de los comisionistas y la especulación de los precios se organizan como representantes de lo "opaco" para oponerlo a lo "transparente" como bandera de la formalización y la presencia de compañías extranjeras. De qualias específicas y diversas alrededor de lo sucio y lo caliente, la ideología construye cualisignos convencionales que buscan organizar la distribución de recursos.

\section{La ideología semiótica del proceso de formalización}

Webb Keane (2003) define ideología semiótica como el conjunto de "suposiciones básicas acerca de lo que son los signos y acerca de cómo funcionan en el mundo” (419). Las ideologías semióticas no son solo sobre signos sino sobre su constitución práctica, sobre lo que hacen en las personas y lo que ellas hacen alrededor de ellos. El régimen de valor de la formalización constituye un sistema de lectura de signos específicos — unas manos sucias, el nombre propio de un comerciante en el Rucom, un microscopio del CDTEC - a partir de la oposición de cualidades que, luego, aplicadas a otros sujetos y otras prácticas, buscan definir su lugar en el momento actual del mercado.

Hay una fotografía particularmente representativa de la oposición sucio/ limpio. La encontré en la cuenta de Instagram de District Itoco, una pequeña compañía de explotación y comercio de esmeraldas en Colombia que funciona desde el 2014 y que tiene sede en Nueva York, Toronto y Bogotá. La escena de la imagen es reiterativa en el mundo de las piedras preciosas: en un primer plano aparece una mano sucia sosteniendo una esmeralda en bruto; en segundo plano se ve parte del dorso de la mano de un hombre blanco; luego, un perro negro echado en el suelo terroso de lo que parece ser una casa o un campamento minero y, por último, un casco rojo (podemos suponer que de un minero). Todo es usual, una mano sucia del minero sostiene una esmeralda. Pero lo que no es usual es el comentario que acompaña la fotografía: "Excuse the dirty hands but the life that pops from even a low quality Colombian emerald is breathtaking. \#Jewelry 
\#Rough \#Emeralds"9 (District Itoco 2015). La persona que sostiene la esmeralda está sucia. Igual que Hilda, enmarca su suciedad en la oposición sucio/limpio, pero esta vez para ubicarse de manera distinta. Y lo hace a través de una figura retórica concreta, las excusas. En un ensayo dedicado al papel de las excusas como fuente para la comprensión de la ética, el filósofo inglés John Austin (1956) dice que

[...] examinar excusas es examinar casos en los que hay algo anormal o en los que algo ha fallado: y, como ocurre a menudo, lo anormal otorga luz a lo normal, su examen nos ayudará a penetrar el velo cegador de la facilidad y la obviedad que esconde los mecanismos de un suceso exitoso. (5-6)

Podemos preguntar, entonces: ¿qué fallo reflejan las excusas del comerciante extranjero?, ¿en qué condiciones se da ese fallo y en qué condiciones las excusas hacen de su acto un acto exitoso? Al excusarse, el comerciante evoca tres dimensiones semióticas distintas, la materialidad de la esmeralda, un lenguaje de valor específico que permite hablar de "la vida" de la esmeralda (la combinación entre transparencia y color) y otro en el que tiene lugar la oposición sucio/ limpio. Al pedir excusas, el comerciante no solo deja claro su acto fallido (haberse ensuciado), no solo lo repara (con las excusas), sino que, ante todo, hace explícita la convivencia de los dos lenguajes de valor en los que el fallo y la reparación tienen lugar: aquel que metafóricamente les otorga "vida” a las esmeraldas -y que justificó que decidiera ensuciarse - y aquel en el que la oposición sucio/limpio es importante -y que justifica que pida disculpas-. De nuevo, la experiencia sensorial de estar sucio es evaluada y, al mismo tiempo, sirve para evaluar. Pero la imagen nos ayuda a ver algo todavía más importante, la oposición sucio/ limpio, y en general la ideología, no es una fórmula fija que permita etiquetar a las personas. Más bien, sugiero, es un recurso simbólico que se evoca de maneras flexibles en prácticas como la del comerciante; a pesar de declarar su fallo, el comerciante había decidido fallar voluntariamente, había decidido ensuciarse. Si nos imaginamos la situación en que lo hizo — seguro, la exploración en un túnel minero-, es posible suponer que estar sucio allí no resultaba un acto fallido sino todo lo contrario, uno exitoso: entrar a una mina, soportar las altas temperaturas, esforzar la vista, picar la roca, etc.; de igual manera, pedir excusas en un nuevo lenguaje de valor es lo que posteriormente le resulta exitoso. El ejemplo es útil para pensar que los actores se apropian de manera creativa - aunque no

9 "Disculpe las manos sucias, pero la vida que emerge de una esmeralda colombiana, incluso siendo de baja calidad, deja sin aliento. \#joyería \#enbruto \#esmeralda". 
del todo libre pues, en últimas, no todos pueden colgar imágenes en Instagram ni hablar inglés - de una oposición que pareciera fija, para moverse entre regímenes de valor distintos. Ideológicamente, sin embargo, resulta útil presentar esta oposición como fijada, como dotada de cierta naturalidad que le permite, como si fuese inevitable, organizar a los sujetos. Esta es otra de las características de las ideologías semióticas, se constituyen a partir de lo que Miyako Inoue (2004) llama inversión indexical (43): la ideología opera creando un sistema semiótico que emerge de experiencias particulares, para luego, en el proceso de convencionalización, hacer que las experiencias originales y otras nuevas parezcan emerger de él. Por medio del despliegue de prácticas particulares orientadas así, la relación entre lo indicado y lo indexical se invierte y el sistema de organización pareciera cubrirse de naturalidad, de inevitabilidad. Un fragmento de la entrevista que sostuve con Gregory Roush en su casa (experimentado exportador y antiguo empleado del GM), es diciente acerca de esta aura de inevitabilidad de la ideología:

La formalización es una cosa que tarde o temprano tenía que aparecer. La llegada del futuro al gremio de las esmeraldas. Tú ya sabes que el gremio está arraigado en el pasado, en las tradiciones pasadas que hoy en día casi no tienen significación. Realmente es muy artesanal y fotogénico y colorido y agradable mirar la manera antigua como se hacía minería, talla e intercambio, pero en realidad no es el futuro, y ya llevamos dieciséis años del nuevo milenio, iy por fin estamos llegando a formalizar esto! (entrevista, febrero del 2016; énfasis añadido)

En no pocas ocasiones he leído y escuchado referencias a un pasado sucio, a un futuro limpio y a la transición como un proceso lineal e inevitable: "la llegada del futuro", limpiar las "manchas del negocio" (Fishbane 2005), la "reputación turbia” y la "imagen desteñida" (Panoussian 2015). Si con Elizabeth Ferry (en prensa) entendemos el valor como la "política de crear y clasificar diferencias y decidir cuáles son importantes”, podemos decir que la limpieza y la suciedad aparecen como directrices de valor, es decir, como diferencias que importan. Lo que prácticas como las de Hilda o las del comerciante extranjero, entre muchos otros, nos muestran es la relatividad de la naturalización ideológica de "la llegada del futuro", una ideología en la que lo sucio y lo limpio se convierten en convenciones, en cualisignos que permiten ordenar el escenario actual del mercado.

Algo similar ocurre con la oposición caliente/frío. Como vimos, la fiebre verde puede ser entendida como una metáfora que se hace literal en distintas experiencias de la economía esmeraldera y cuyo poder enunciador ha entrado en decadencia con el aumento de la escasez. Pero existe otra transformación en 
la que hablar de la fiebre verde ya no resulta pertinente o, más bien, lo resulta para distanciarse de ella: la fiebre verde representa la tradición minera, lo arcaico, la pasión, la obsesión, las fábulas, el pensamiento obnubilado producto de los calores infernales de los túneles clandestinos; todo lo contario a lo que ideológicamente se plantea como un mercado "frío". Cuando en alguna ocasión quise hablar de las historias de las guerras esmeralderas con un representante del gremio ante el Estado, su respuesta fue diciente: "Siempre procuro no hablar de eso, porque ya no viene al caso [...] Es mejor bajarles la temperatura a esas cosas. No hablarlo tanto" (Alberto Acuña, entrevista, febrero del 2016). De lo mismo me hablaba un comerciante bogotano que trabaja con dos socios estadounidenses vendiendo esmeraldas y especímenes minerales. En una entretenida anécdota describía la búsqueda afiebrada e ilusoria de los mineros y contaba cómo intentaba convencer a sus socios gringos de no meterse en esos "asuntos calientes" sino, más bien, pensar con "cabeza fría” como, según él, hacían los pocos inteligentes metidos en el negocio de las esmeraldas (Javier Benavides, entrevista, agosto del 2015). Frente al comercio caliente está el comercio desapasionado, frío, inteligente; están los certificados de las piedras a los que Ronald Ringsrud se refiere como documentos "fríos" en la que es quizá la fuente más completa sobre los detalles de la explotación y el comercio de esmeraldas en Colombia (2013, 206); está un futuro sin eventos violentos, un futuro que deje atrás el pasado caliente; están las nuevas tecnologías de MTC para enfriar las minas. Si la ideología de la formalización puede ser vista como un proceso de limpieza, también puede serlo como uno de enfriamiento: de las minas, de las cabezas, de la temporalidad misma de la economía esmeraldera, de la explotación y del comercio en general.

Existe una convención cuyo poder enunciativo, cuya capacidad para actuar y para orientar la acción es todavía más profundo, la oposición entre lo transparente y lo opaco. Igual que la oposición sucio/limpio, esta oposición está relacionada con la materialidad de las esmeraldas y con experiencias sensoriales concretas ordenadas en un régimen de valor particular; se habla de esmeraldas más o menos transparentes, de mercados más o menos transparentes, de Estados más o menos transparentes, de personas transparentes y de personas opacas (Ballestero 2012; West y Sanders 2003). En el marco ideológico de la formalización, la oposición efectivamente tiene un papel central en la distribución de recursos a la vez que, al igual que las otras, puede entenderse como un recurso en sí misma. En efecto, desde hace unos años, la transparencia se ha convertido en el valor central de la transformación de la economía esmeraldera. Encarnada en la trazabilidad (y en documentos como el Rucom y el Certificado de Origen), la transparencia busca hacer que todo sea visible y, particularmente, 
que lo sean la circulación de personas y de objetos - esmeraldas en este caso-. El papel de la transparencia en el momento actual nos ayuda a pensar dos asuntos, el carácter visual de la explotación y el comercio de las piedras, y su oposición a lo opaco como cualidad en la que se conjugan muchas de las prácticas de la tradición esmeraldera en Colombia.

Todos tenemos en nuestra cabeza la imagen de alguien que ve una esmeralda a trasluz. Podemos ver a esa persona sosteniendo la piedra entre sus dedos pulgar e índice, elevándola hacia el sol, cerrando un ojo y dándole vueltas para poder observarla desde todos los ángulos. Lo hacen mineros boyacenses, lo hacen comerciantes bogotanos, lo hacen compradores en Manhattan y en joyerías del centro de Bogotá. En este habitual y simple gesto se esconde uno de los ejes centrales que permite acercarse a la comprensión del comercio y explotación de esmeraldas en Colombia, el acto de ver las esmeraldas. Saber verlas es el piñón sobre el que se monta toda la maquinaria alrededor de las piedras. Más aún, esta visibilidad trasciende la experiencia misma de ver las piedras y se extiende a toda la economía. Todo el mercado pareciera moverse alrededor del conflicto visual sobre lo que se ve, lo que no se ve y sobre quiénes pueden ver qué. El proceso mismo de formalización, quiero sugerir, puede ser entendido como un conjunto de mecanismos para que los representantes del Estado y del GM —que, según me enteré, trabaja en un software que garantice la trazabilidad-lo vean todo. En este sentido, el proceso de formalización y la presencia del GM han implicado un reacomodo de los regímenes de visibilidad (Crary 2008), no solo en cuanto a la aparición de nuevas herramientas visuales (desde las cámaras que ahora inundan las minas de MTC hasta los microscopios y los documentos mismos de la formalización), sino en cuanto a la distribución misma de lo que vale la pena ver y de quiénes pueden ver qué. En este reacomodo de las coordenadas visuales, la oposición transparente/opaco ha comenzado a ser construida como un recurso simbólico central a partir de documentos especializados sobre gemología, revistas especializadas de comercio de gemas y declaraciones públicas de las compañías mineras extranjeras (pequeñas y grandes). Con los años, y al compás de la transparencia como la varita mágica para garantizar la transición hacia la modernización en todo el mundo (West y Sanders 2003), esta oposición suele ser evocada de manera permanente para hablar de sujetos, de espacios y de prácticas específicas en la economía esmeraldera. Así, el uso de apodos en lugar de los nombres propios se convierte en la representación de una lógica oculta; la densidad de la red de intermediarios es vista como una dificultad en los intentos de transparentarlo todo; el regateo permanente en los precios dificulta lo que se asume como algo natural: el precio como representación del valor. Todas estas 
prácticas han comenzado a empaquetarse en torno a la cualidad de lo "opaco". Igual que de un mercado sucio o caliente, suele hablarse de un "negocio opaco" (Palomino y Lafuente 2016), de comerciantes cuya "idiosincrasia" hace que les "guste vivir en las sombras" (Alberto Acuña, entrevista, febrero del 2016) o que "no quieren que haya evidencia de nada" (Luisa Acosta, entrevista, febrero del 2016). En contraposición, se habla de un "comercio más transparente" ("Esmeraldas, al microscopio" 2012) y de políticas de "transparencia y honestidad" (Fedesmeraldas 2016). Las páginas web de las empresas, los reportes de prensa, las entrevistas públicas de los funcionarios del Estado, los documentos públicos, todos se llenan de la palabra transparencia y de su oposición a gran parte de la tradición: el pasado sucio, "las vetas rojas" del pasado (Blanco 2015), las "reglas arcaicas”, la fiebre verde, los patrones esmeralderos, la bravura, el derroche, el consumo desmesurado de alcohol, las viejas técnicas de talla.

Esta transparencia se ha convertido, entonces, en una suerte de "caja negra”, en una red de "actores que se ha estabilizado hasta el punto de que sus infraestructuras de asociación no necesitan ser explicadas o aun entendidas, de manera que actúan en el mundo como objetos que son dados por hecho" (Ferry, en prensa, 32). En la transparencia como convención opera una doble relación frente al fetichismo de las mercancías. Por un lado, y hasta cierto punto, la idea de transparencia, encarnada en la de trazabilidad, ha tenido origen en un intento por poner en cuestión el fetichismo alrededor de las piedras preciosas, por hacer visible parte de los procesos de extracción, producción y comercialización de, en este caso, las esmeraldas. Sin embargo, por otro lado, la solución ha sido, si se quiere, un nuevo proceso de fetichización: la transparencia y la trazabilidad se convierten en convenciones que, en apariencia, automáticamente garantizan la producción y distribución "justa” de las mercancías pero cuyas condiciones y procesos de producción no suelen ponerse en cuestión. Lo que he mostrado en este documento permite comprender que detrás de la transparencia-de-la-transparencia está el despliegue de documentos específicos, de modos específicos de contabilizar (y, para ello, de concebir) a las personas, de categorías específicas para ordenar que, siempre, implican conflictos particulares y que (como en cualquier proceso hegemónico) están en permanente construcción (Ballestero 2012).

Sin embargo, de nuevo, a pesar de su naturalidad, resulta más útil leer la oposición opaco/transparente como un recurso disponible para su evocación en prácticas determinadas. Las sospechas que recorren el momento actual de la economía son útiles para ello. Los ejemplos son múltiples, superan los objetivos 
de este artículo, pero son un diagnóstico de la complejidad de esa aparente temporalidad lineal de la ideología. Así, por ejemplo, el GM se mueve permanentemente entre la transparencia como bandera y la opacidad como estrategia de funcionamiento. Por ello, para muchos en el mercado, es un actor más oculto que visible, se tejen especulaciones acerca de si es una empresa gringa, canadiense o mexicana; se especula sobre si pagan impuestos o no; se rumora que exportan las piedras en bruto y no talladas y que por eso le pagan menos dinero al Estado; se cuentan historias de la relación entre sus directivas y las fuerzas militares de Estados Unidos; sus oficinas, de hecho, están en la zona financiera de la Avenida Chile en Bogotá, lejos de los lugares tradicionales de transacción en las calles del centro de la ciudad, en donde lo que vale es estar presente, conocer los rostros, verse. Cuando en algún momento llamé a las oficinas de MTC para pedir una cita, me respondieron que eran una empresa muy "discreta” y que no estaban autorizados a brindar entrevistas académicas. Algo similar ocurre con los documentos de registro de la formalización minera, pocos los entienden y muchos sospechan de ellos; asumen que el cobro de impuestos terminará por meterlos en un lenguaje financiero al que simplemente no están acostumbrados, que no entienden y que ni siquiera logra reflejar las particularidades del comercio de esmeraldas que lo diferencian de, por ejemplo, el del oro. El mismo uso de los nombres propios como requerimiento para el registro resulta para ellos contrario al reconocimiento que durante décadas han dado los apodos; es decir, los documentos de la transparencia son, para ellos, más bien prácticas de opacidad.

Con todo, las sospechas alrededor de los documentos de registro y alrededor del GM y de la formalización en general no giran solamente en torno a un actor o a un documento; ni siquiera pueden reducirse a las sospechas acerca de las “verdaderas intenciones” de los abanderados de la formalización. Las sospechas permanentes cuestionan no solo los signos de "lo opaco" sino el sistema mismo de significación, la ideología misma que, literalmente, pone en riesgo el modo de subsistencia de lo que varios comerciantes llaman la "cadena productiva de las esmeraldas”. La construcción de prácticas como signos definidos en un sistema semiótico particular representa un fenómeno mucho más abierto que la inevitable línea entre lo sucio y lo limpio, lo caliente y lo frío, y lo opaco y lo transparente. Representan algo mucho más complejo que la simple "llegada del futuro". 


\section{Referencias}

Austin, John. 1956. “A Plea for Excuses: The Presidential Address”. Proceedings of the Aristotelian Society 57: 1-30.

Ballard, Chris y Glenn Banks. 2003. “Resource Wars: The Anthropology of Mining”. Annual Review of Anthropology 32: 287-313.

Ballestero, Andrea. 2012. “Transparency in Triads”. PoLAR 35 (2): 160-166.

Baquero, Petrit. 2017. La nueva guerra verde. Bogotá: Planeta.

Barrena, Sara. 2014. “El pragmatismo”. Factótum 12: 1-18. http://www.revistafactotum.com/ revista/f_12/articulos/Factotum_12_1_Sara_Barrena.pdf.

Blanco, Mario. 2015. “MTC, regresa esperanza a industria verde”. Mundo Minero. Consultado el 16 de agosto del 2016. http://mundominero.com.co/mtc-regresa-esperanza-a-industriaverde/.

Brazeal, Brian. 2014. "The History of Emerald Mining in Colombia: An Examination of Spanish-Language Sources”. Extractive Industries and Society 1 (2): 273-283.

-. 2015. "Central Asian Crypto-Jews in the Global Emerald Economy”. Inédito.

-. 2016. "Nostalgia for War and the Paradox of Peace in the Colombian Emerald Trade". EXtractive Industries and Society 3 (2): 340-349.

Calvão, Filipe. 2013. "The Transporter, the Agitator, and the Kamanguista: Qualia and the Invisible Materiality of Diamonds”. Anthropological Theory 13 (1/2): 119-136.

Carr, Summerson y Michael Lempert, eds. 2016. Scale: Discourse and Dimensions of Social Life. Oakland: University of California Press.

Chumley, Lily. 2013. "Evaluation Regimes and the Qualia of Quality”. Anthropological Theory 13 (1-2): 169-183.

Chumley, Lily y Nicholas Harkness. 2013. "Introduction: Qualia”. Anthropological Theory 13 (1-2): 3-11.

Claver, Pedro. 1993. La guerra verde. Treinta años de conflicto entre los esmeralderos. Bogotá: Intermedio.

Crary, Jonathan. 2008. Las técnicas del observador. Visión y modernidad en el siglo XIX. Murcia: Cendeac.

Dessay, Charles Mathieu. 2011. "El mito de Muzo se derrumba”. El Espectador, 23 de abril. Consultado el 16 de agosto del 2016. http://www.elespectador.com/noticias/economia/elmito-de-muzo-se-derrumba-articulo-264688.

District Itoco. 2015. “@districtitoco: Excuse the dirty hands but the life that pops from even a low quality Colombian emerald is breathtaking”. Publicación de Instagram. Consultado el 16 de agosto del 2016. https://www.instagram.com/districtitoco/.

“Esmeraldas, al microscopio". 2012. Mundo Minero, 18 de octubre. Consultado el 16 de agosto del 2016. http://mundominero.com.co/esmeraldas-al-microscopio/. 
Fedesmeraldas. 2016. “Mothergem”. Consultado el 7 de junio del 2016. http://mothergem.co.

Fehérváry, Krisztina. 2013. Politics in Color and Concrete. Social Materialities and the Middle Class in Hungary. Indiana: Indiana University Press.

Ferry, Elizabeth. 2011. No solo nuestro. Patrimonio, valor y colectivismo en una cooperativa guanajuatense. Zamora, Ciudad de México: El Colegio de Michoacán; Universidad Iberoamericana.

-. 2016a. "Gold Prices as Material-Social Actors: The Case of the London Gold Fix". The Extractive Industries and Society 3: 82-85.

—. 2016b. “On Not Being a Sign: Gold’s Semiotic Claims”. Signs and Society 4 (1): 57-79.

-. En prensa. Minerales, coleccionismo y valor en la frontera México-Estados Unidos. Ciudad de México: El Colegio de Michoacán. Originalmente publicado como Minerals, Collecting, and Value across the U.S.-Mexican Border. 2013. Bloomington: University of Indiana Press.

Ferry, Elizabeth y Mandana Limbert, comps. 2008. Timely Assets. The Politics of Resources and their Temporalities. Santa Fe: School for Advanced Research Press.

Fishbane, Matthew. 2005. "New Luster for Colombia's Gem Industry”. The Christian Science Monitor, 21 de junio. Consultado el 26 de agosto del 2016. http://www.csmonitor.com/2005/ 0621/p06s01-woam.html.

Gal, Susan. 1989. "Language and Political Economy”. Annual Review of Anthropology 18: 345-367.

-. 2013. "Tastes of Talk: Qualia and the Moral Flavor of Signs”. Anthropological Theory 13 (1/2): 31-48.

Geertz, Clifford. 1978. "The Bazaar Economy: Information and Search in Peasant Marketing”. American Economic Review 68 (2): 28-32.

Guerrero, Javier. 1985. "La economía esmeraldífera y la violencia: la microhistoria institucional y contrainstitucional”. Memorias del V Congreso de Historia, 227-247. Serie de Memorias de Eventos Científicos. Armenia: Universidad del Quindío; Icfes.

Gutiérrez, Francisco y Mauricio Barón. 2008. "Órdenes subsidiarios. Coca, esmeraldas: la guerra y la paz”. Colombia Internacional 67: 102-129.

Harkness, Nicholas. 2013. “Softer soju in South Korea”. Anthropological Theory 13 (1-2): 12-30.

-. 2014. Songs of Seoul. An Ethnography of Voicing in Christian South Korea. Berkeley: University of California Press.

-.2015. “The Pragmatics of Qualia in Practice”. Annual Review of Anthropology 44 (1): 573-589.

Inoue, Miyako. 2004. "What Does Language Remember?: Indexical Inversion and the Naturalized History of Japanese Women”. Journal of Linguistic Anthropology 14 (1): 39-56.

Keane, Webb. 2003. "Semiotics and the Social Analysis of Material Things". Language and Communication 23 (3-4): 409-425.

Kockelman, Paul. 2006. "A Semiotic Ontology of the Commodity". Journal of Linguistic Anthropology 16 (1): 76-102. 
-. 2016. The Chicken and the Quetzal. Incommensurate Ontologies and Portable Values in Guatemala's Cloud Forest. Durham: Duke University Press.

Leiteritz, Ralf y Manuel Riaño. 2016. “Tras el corazón verde: los vaivenes del conflicto en la región esmeraldera de Colombia”. Consultado el 16 de agosto del 2016. https://www. academia.edu/23057980/Tras_el_coraz\%C3\%B3n_verde_los_vaivenes_del_conflicto_en_ la_regi\%C3\%B3n_esmeraldera_de_Colombia.

Maurer, Bill. 2006. “The Anthropology of Money”. Annual Review of Anthropology 35: 15-36.

"Minería Texas Colombia se pronuncia sobre la invasión de mina de esmeraldas en Boyacá”. 2015. RCN Radio, 22 de mayo. https://www.rcnradio.com/colombia/mineria-texascolombia-se-pronuncia-sobre-invasion-de-mina-de-esmeraldas-en-boyaca

Munn, Nancy. 1986. The Fame of Gawa. A Symbolic Study of Value Transformation in a Massim Society (Papua New Guinea). Durham: Duke University Press.

Palomino, Sally y Javier Lafuente. 2016. "Esmeraldas: un negocio popular y opaco en Colombia”. El País, 14 de mayo. Consultado el 16 de agosto del 2016. http://internacional.elpais.com/internacional/2016/05/14/colombia/1463185065_658956.html.

Panoussian, Florence. 2015. "Las esmeraldas de Colombia limpian su reputación”. Consultado el 16 de agosto del 2016. https://es.noticias.yahoo.com/las-esmeraldas-colombia-limpian-su-reputaci\%C3\%B3n-161533999.html?guccounter=1.

Páramo, Carlos. 2011. "El corrido del minero: hombres y guacas en el occidente de Boyacá". Maguaré 25 (1): 25-109.

Parra Bautista, Johanna. 2006. "Familia, poder y esmeraldas. Relaciones de género y estructura económica minera en el occidente de Boyacá, Colombia”. Revista Colombiana de Antropología 42: 15-53. http://www.scielo.org.co/scielo.php?script=sci_arttext\&pid $=$ S0486-65252006000100002.

Pérez, Vanessa. 2015. "La cena del Grupo Muzo Emerald Colombia en Bogotá". Jet-Set, 21 de diciembre. Consultado el 16 de agosto del 2016. http://www.jetset.com.co/sociedad/ bogota/galeria/la-cena-del-grupo-muzo-esmerald-colombia-en-el-museo-botero-en-bogota/142846.

Pineda, Jacinto. 2008. "Territorio y poder: caso zona esmeraldífera occidente de Boyacá. 1960-1973”. Tesis de Maestría en Historia, Facultad de Ciencias de la Educación, Universidad Pedagógica y Tecnológica de Colombia, Tunja.

Piñeros, Robinson y Luz Herrera. 2004. "La violencia en el occidente de Boyacá: el conflicto esmeraldero, evolución reciente 1990-2002”. Tesis de Licenciatura en Ciencias Sociales, Facultad de Ciencias Sociales, Universidad Distrital Francisco José de Caldas, Bogotá.

"Polémica tras la venta más grande de Carranza”. 2015. Semana, 23 de mayo. Consultado el 16 de agosto del 2016. http://www.semana.com/nacion/articulo/la-polemica-tras-la-ventade-la-mina-mas-grande-de-carranza/428750-3\#.

Restrepo, Mariluz. 1990. "La semiótica de Charles S. Peirce”. Signo y Pensamiento 16: 27-46.

Restrepo, Pedro, Adolfo Polo y Édgar Mayorga. 1996. “Conflicto esmeraldífero en el occidente de Boyacá”. Tesis de licenciatura, Pontificia Universidad Javeriana. 
Ringsrud, Ronald. 2013. Esmeraldas: una guía apasionada. Las esmeraldas, la gente, sus secretos. Bogotá: Maremagnum.

Silverstein, Michael. 1976. "Shifters, Linguistic Categories, and Cultural Description”. En Meaning in Anthropology, editado por Keith Basso y Henry Selby, 11-55. Albuquerque: University of New Mexico Press.

Torres, Andrés y Antonio Ordóñez. 1995. “Conflicto y pacificación en el occidente de Boyacá. 1970-1995”. Tesis de Maestría en Ciencia Política, Departamento de Ciencia Política, Universidad de los Andes, Bogotá.

Uribe, María Victoria. 1992. Limpiar la tierra. Guerra y poder entre esmeralderos. Bogotá: Cinep.

Valentin, Daniel. 1996. Charred Lullabies. Chapters in an Anthropography of Violence. Nueva Jersey: Princeton University Press.

Vega, Khaaterine. 2015. "Primera marcha del paro de pequeños y medianos mineros, ¿continuará?”. Desde Abajo, 19 de febrero. Consultado el 16 de agosto del 2016. https://www. desdeabajo.info/colombia/item/25888-primera-marcha-del-paro-de-pequenos-y-medianos-mineros-continuara.html.

Walsh, Andrew. 2004. "In the Wake of Things: Speculating in and about Sapphires in Northern Madagascar”. American Anthropologist 106 (2): 225-237.

West, Harry y Todd Sanders, comps. 2003. Transparency and Conspiracy: Ethnographies of Suspicion in the New World Order. Durham: Duke University Press.

\section{Entrevistas citadas}

Alberto Acuña, febrero del 2016.

Confedesmeraldas, entrevista colectiva (Pedro Otálora, Agustín Camacho e Hilda Cruz), marzo del 2016.

Gregory Roush, febrero del 2016.

Javier Benavides, agosto del 2015. 